\title{
Nuevas subjetividades: una aproximación posthumanista y material a los procesos de encuentro entre niñas, niños y psicoestimulantes
}

\author{
New subjectivities: a posthumanist and neo- \\ materialist approach to the processes of \\ encounter between children and psychostimulant
} medication

\section{Novas subjetividades: uma abordagem pós- humanista e material dos processos de encontro entre meninas, meninos e psicoestimulantes}

Sebastián Rojas Navarro ORCID ID: 0000-0003-1963-1569 Centro de Justicia Educacional, Pontíficia Universidad Católica de Chile, Chile

Autor referente: srojan@uc.cl

Historia Editorial

Recibido: 20/04/2018

Aceptado: 04/10/2018

\section{RESUMEN}

Este artículo propone un abordaje complejo y posthumanista a los diversos modos mediante los cuales niñas, niños y psicofármacos se coafectan, determinando diversos devenires ontológicos a partir de esta interacción, en el nicho provisto por dos escuelas ubicadas en la zona oriente de Santiago de Chile. A partir de los resultados de una praxiografía que se extendió por ocho meses con niñas y niños de 9 y 10 años, se sostiene que es necesario pensar esta relación entre niñas, niños y psicofármacos desde coordenadas distintas a las provistas por las tesis de la medicalización. Esto, en tanto ellas reducen y achatan la noción de subjetividad a una posesión interna del individuo, y a una separación radical con el mundo, sostenida en una discutible idea de excepcionalismo humano. En respuesta a esta idea de subjetividad, se propone otra que permite un abordaje diferente al problema, en tanto es una subjetividad entendida en 
sus aspectos procesales, dinámicos, y últimamente sostenido en base a entramados con el mundo y con la medicación.

Palabras claves: Posthumanismos; Entramados farmacológicos; Niñas y niños; Psicoestimulantes

\section{ABSTRACT}

This article advances a posthumanist and complex approach to the different ways in which children and psychostimulants can co-affect each other, enabling different ontological becomings thanks to the processes of coming together that took place in the niche provided by two schools located in East Side of Santiago, Chile. Grounded in the results of an 8-months praxiography with children aged 9 and 10 -years old, I argue for the need of new coordinates to reflect upon these interactions, coordinates different to those granted by the medicalization thesis. This, inasmuch as their approach tends to flatten and reduce the subjectivity of the medicated child to an inner essence, radically separated from the world, which is sustained under a disputed idea of human expectionalism. In respond to this, I advance an idea of subjectivity which is relational, processual and sustained on entanglements with the world and with the medication.

Keywords: Posthumanism; Pharmaceutical entanglements; Children; Subjectivity

\section{RESUMO}

Este artigo propõe uma abordagem complexa e pós-humanista aos vários modos pelos quais meninos, meninas e psicofármacos se co-afetam, determinando diferentes devires ontológicos dessa interação, no nicho prestado por duas escolas localizadas na zona oriente de Santiago do Chile. A partir dos resultados de uma praxiografía que durou oito meses com meninas e meninos com idade entre 9 e 10 anos, argumenta-se que é necessário pensar sobre a relação entre crianças e psicofármacos a partir de coordenadas diferentes das previstas pelas teses da medicalização. Neste aspecto, estas teses reduzem e achatam a noção de subjetividade a uma posse interna do indivíduo, e uma separação radical do mundo, realizada em uma ideia discutível do excepcionalismo humano. Em resposta a esta idéia de subjetividade, propõe-se uma abordagem diferente para o problema, a partir de uma subjetividade entendida em seus aspectos processuais, dinâmicos e, finalmente, sustentada com base nas associações com 0 mundo e com a medicação.

Palavras chave: Pós-humanismos; Associações farmacológicas; Meninas e meninos; Subjetividade 
Indeed, how might the contemporary social sciences come to address this buzzing world whereby all the modern demarcations that found their practices and conventional objects of study have been meshed up in relations with entities and practices that they thought belong to an entirely different realm? How are they to take up the adventure of relevance, of entertaining the question of 'how it is, here, that things matter?', when their modern ethics of inquiry hardly prepare them for the heterogeneous, naturalcultural encounters that the question is likely to generate? How might they invent a manner of encountering worlds that matters? (Savransky, 2016. pp. 47-48)

\section{Nuevas formas de pensar viejas preguntas: pensar subjetividades posthumanas}

urante las últimas décadas, una serie de transformaciones teóricometodológicas han impulsado una profunda revisión de muchos de los presupuestos de las ciencias sociales, cuestionando su capacidad de dar cuenta de las complejidades del mundo actual. Esto, en tanto se acusa que la herencia profundamente moderna de las ciencias sociales ha teñido gran parte de sus conceptos y teorías de un fuerte humanismo y antropocentrismo, elementos que parecen tornarse obsoletos a la hora de pensar los modos actuales de configuración y reconfiguración del mundo social. Braidotti (2016) esquematiza este nuevo ethos emergente de las ciencias sociales y humanas del siguiente modo:

En gran medida una filosofía del exterior, de los espacios abiertos y de enacciones corporizadas [embodied enactments], el pensamiento posthumano anhela por un salto cualitativo fuera de lo familiar, confiando en las posibilidades inexploradas que se abren por nuestra localización histórica en el mundo tecnológicamente mediado de hoy en día. Es una forma de ser digna de nuestro tiempo el incrementar nuestra libertad y comprensión de las complejidades que habitamos en un mundo que no es ni antropocéntrico ni 
antropomórfico, sino más bien geopolítico, ecosófico y orgullosamente zoécentrado. (p. 27)

El nuevo ethos de las ciencias sociales ha sido orientado por una serie de giros, los cuales han introducido nuevas formas de problematizar y conceptualizar tanto al mundo social como a las interconexiones y articulaciones que se establecen entre éste y los individuos (por ejemplo ver Barad, 2003; Coole \& Frost, 2010; Mol, 1999). Argumentando una falta de imaginación para problematizar el mundo actual por parte de los enfoques tradicionales, estas nuevas aproximaciones se distancian de sus antecesores a la vez que claman por nuevas comprensiones que destaquen el carácter siempre cambiante del mundo social-en tanto atravesado por transformaciones económicas, sociales, políticas y ecológicas-lo cual constantemente conlleva resultados imprevistos que demandan nuevas conceptualizaciones, así como formas novedosas de reflexión sobre el mismo (Savransky, 2016). La relevancia de estos giros reside entonces en su originalidad teórico-metodológica, la cual permite elaborar nuevas preguntas de investigación, y nuevas formas de interrogar el(los) mundo(s) social(es) donde cotidianamente se juega nuestra experiencia, resaltando así su carácter innovador, complejo y contingente.

Un tema que ha sido reexaminado a la luz de los posthumanismos es la pregunta respecto a la subjetividad. Frente a las conceptualizaciones postuladas por los enfoques tradicionales que sostienen una aproximación a la subjetividad tomada por dicotomías y binarismos propiamente modernos y humanistas-por ejemplo, ¿es la subjetividad producida externamente, o es ésta una propiedad interna del individuo?_los posthumanismos proponen una idea de la subjetividad en donde ésta es conceptualizada como un fenómeno complejo y procesal, producido en una relación de co-afectación con el mundo, en donde la subjetividad sería una estabilización transitoria de una serie elementos heterogéneos, que no serían ni propiamente 
internos ni externos al individuo. Más que definirse por su carácter identidatario y autocentrado, aparece una visión nómade del individuo (Braidotti, 2016), la cual enfatiza su caracter local y sus dinámicas de corporización, así como también su configuración relacional y afectiva. La subjetividad es entendida como siempre emergente, y estabilizada temporalmente gracias $a-y$ en consecuendia de-las relaciones que se establecen con un cosmos entendido como fundamentalmente procesal y carente de jerarquias prefijadas, un cosmos marcado por las diversas formas entramadas de existencia que en el cohabitan, componiéndolo (Latour, 2004; Stengers, 2011). Así, esta nueva conceptualización de la subjetividad abandona la tradicional idea de una interioridad, en tanto que la subjetividad no sería una propiedad discreta del individuo, ni estaría separada de lo naturalcultural. Por lo tanto, una aproximación posthumanista deberá evitar reducir la subjetividad a un epifenómeno o residuo de naturaleza o cultura (Rojas, 2017).

Este énfasis en lo procesal de la subjetividad, así como en su composición heterogénea, posibilita pensar de modos productivos los procesos de encuentro entre entidades de diverso orden. Productivos, en tanto cada encuentro produce una modificación-pero no necesaria ni exclusivamente una supresión-de aquellos elementos involucrados en dicho proceso. Un encuentro no es únicamente una reunión de elementos_-por ejemplo, un niño y un fármaco-sino que es un proceso de transformación, de devenir conjunto, el cual puede producir una nueva forma de asociatividad:

De esta forma, el encuentro mismo, cuando es exitoso, puede producir una nueva sociedad—por ejemplo, puede producir una emoción, una proposición, o un niño—que puede, a su vez, llegar a disfrutar su propia historia y encontrarse con otras entidades y nichos. (Savransky, 2016. p. 94)

Esta apertura a pensar la subjetividad desde nuevas coordenadas permite examinar, con una mirada renovada, los procesos de encuentro y transformación que se 
producen en el nicho provisto por la sala de clases, cuando estas entidades que se topan son niñas, niños y psicofármacos. La necesidad de volver a examinar estas dinámicas se funda en la imposibilidad de seguir achatando y simplificando estos fenómenos propios de un mundo complejo, a través de explicaciones universalistas y desancladas de las prácticas concretas mediante las cuales estas entidades se encuentran. Principalmente, permiten dar una mirada alternativa a aquella que proveen las tesis de la medicalización de la infancia (Conrad \& Schneider, 1992).

\section{Trastorno por déficit de atención/hiperactividad (TDAH), psicofármacos y teorías} de la medicalización de la infancia: ¿qué hay más allá?

EI TDAH se ha transformado en diagnóstico rodeado de polémicas, alrededor del cual se han generado diversos debates respecto a su legitimidad y modos de tratamiento, particularmente en relación con abordaje farmacológico (Bergey, Filippe, Conrad \& Singh, 2018). En los últimos años estas polémicas han alcanzado América del Sur, despertando el interés de muchos investigadores por estudiar los procesos y dinámicas mediante los cuales estas discusiones se han trasladado—con diversos grados de variabilidad-a diversas locaciones (Faraone \& Bianchi, 2018).

Desde las ciencias sociales, una de las principales matrices explicativas para reflexionar respecto al incremento de las tasas diagnósticas del TDAH ha sido proporcionada por las tesis de la medicalización. En términos general-y especialmente en sus vertientes más radicales-estas proponen que diagnósticos como el TDAH responden a efectos de control social y legal, regulando comportamientos indeseados o problemáticos exhibidos por niñas y niños en diversas situaciones y contextos. De este modo, los psicofármacos tendrían por fin el normativizar y/o normalizar la subjetividad de aquellos que los consumen, en tanto el fármaco tendría un potencial inherente coercitivo. El uso de psicofármacos entraría en juego como una tecnología del saber biomédico, actuando como un vector de poder 
que tomaría como su objetivo—su blanco-a la niña o niño diagnosticado. De esta operatoria habría únicamente un resultado posible: volver a niñas y niños bajo el control adulto, anulando aquello de esas subjetividades que resista a dicha autoridad, encauzando así el proceso de transformación de niñas y niños para transformarlos en individuos funcionales (Harwood, 2006).

Sin embargo, estas explicaciones en gran medida aplanan la complejidad y dinamismo de la escena. Principalmente, en tanto suelen situar la problemática de la subjetividad de un modo excesivamente simplificado: la niña o niño se encontraría en posesión de una subjetividad, la cual le sería "propia" y estaría depositada "dentro" de sí. Es decir, lo que niñas o niños "son" respondería a propiedades que les son inherentes, las cuales o bien se expresan libremente-sin restricciones—o se encontrarían limitadas, constreñidas por factores externos que entorpecerían su despliegue. En ese sentido, esta consideración de lo humano sostiene que o bien se es capaz de actuar autónomamente, u otros—humanos o no-humanos-actúan sobre la niña o el niño, coartando su capacidad de acción. Que, o bien se es libre y abstinente-es decir, no "influenciado" por sustancia alguna-o se es subyugado por el fármaco, el cual entonces solamente puede ser considerado como destructivo, pero rara vez o nunca como terapéutico (Gomart, 2002).

Gran parte de los estudios realizados bajo la influencia de las tesis de la medicalización de la infancia parten de la moderna suposición de que la producción de subjetividades es efecto de un vector, el cual se imprime sobre ciertos objetos, produciendo formas de existencia predeterminadas y contenidas en el vector en cuestión (Savransky, 2014). La aplicación de un vector con dichas características—tal como el uso de los psicofármacos_tendría consecuencias únicamente restrictivas y disciplinarias, produciendo únicamente un efecto de homogenización de aquello que toma como su objetivo—niñas y niños. Lo curioso de esta formulación es que remueve de la escena la potencialidad de todo encuentro de producir otros efectos, y resta la 
posibilidad de cualquier patrón de difracción a los efectos del vector en cuestión. Es decir, reduce una dinámica de encuentro a un juego de replicaciones y reproducciones, en lugar de mapear sus procesos de interferencia, variabilidad y mutua composición (Haraway, 1992).

Considerando lo anterior, resulta necesario rescatar los nichos en el cuales estas subjetividades están inmersas, así como las variables socio-materiales implicadas en su estabilización, para poder ir más allá de una sobre-simplificación y esquematización que conceptualiza las experiencias de niñas y niños a nivel global a esencialmente el mismo fenómeno, sin importar dónde, cuándo y bajo que con condiciones ocurren estos encuentros, y restando todo valor a las diversas formas de relación que posibilitan diferentes nichos ecológicos entre sus componentes humanos y nohumanos (Rojas Navarro \& Vrecko, 2017). Es precisamente en este punto donde los posthumanismos han posibilitado otra forma de comprender cómo, a nivel micropolítico, estos encuentros posibilitan entramados de relaciones que conllevan formas novedosas, situadas y específicas de co-afectación, de las cuales pueden emerger distintas maneras de habitar y corporizarse a través, y gracias a, estos entramados. Maneras que pueden ser experimentadas como beneficiosas, pero también en modos que pueden experimentarse como desventajosos.

\section{Produciendo entramados farmacológicos: ilustración de encuentros en la sala} de clases

A continuación, discutiré dos escenas que acontecieron durante una praxiografía llevada a cabo en dos colegios de la zona oriente de Santiago, Chile. La praxiografiao estudio de las prácticas-es un tipo de estudio etnográfico que enfatiza los modos en que las prácticas articulan agencias que se encuentran distribuidas entre diversos actores humanos y no-humanos (Mol, 2002). La atribución de agencia a actores nohumanos responde a la intención de trascender la asociación tradicionalmente 
establecida entre agencia, y "las nociones de intencionalidad, racionalidad, y voz". Es decir, con superar la ligazón tradicionalmente establecida entre agencia y "nociones extremadamente limitadas de subjetividad y poder" (Marchand, 2018, pp. 292-293)

En las escenas que discutiré es posible apreciar dos modos distintos en que el encuentro entre diversas entidades-entre ellas una niña, un niño, y los psicofármacos-puede producirse en un contexto determinado. Estas escenas fueron seleccionadas por dos motivos. Primero porque ilustran formas beneficiosas en que dicho encuentro puede darse cuando el proceso de co-afectación es entendido de manera no-binaria en lo referente a la agencia-es decir, que o bien se es agente pese al fármaco, venciéndolo; o se es coactado, y por ende constreñido, sobre-escrito por el fármaco-sino que se entiende la subjetividad como efecto de esta coafectación, y por lo tanto como un elemento emergente que se presenta con el fármaco, en una serie de interacciones y procesos que lo incluyen y posibilitan la emergencia de algo nuevo. Segundo, ya que permiten ver como un entramado puede ser experimentado como desventajoso cuando el efecto del encuentro produce algo nuevo que, pese a su carácter de novedad, se estabiliza como displacentero o adverso. Independientemente del resultado del entramado producido, cabe resaltar que las condiciones de existencia que son posibilitadas por tales entramados son más amplias y matizadas que la reductio ad absurdum descrita por sectores radicales criticando el uso de psicoestimulantes.

Ahora bien, la sobre-simplificación acometida por las teorías de la medicalización no acontece sin cierta justificación. Indudablemente existe cierta precaución con respecto al uso de psicofármacos, la cual se funda en los potenciales riesgos documentados con respecto a su consumo. Considerando esto, se plantea la necesidad de una revisión y supervisión periódica y sostenida de dichos encuentros, precaución establecida por diversos actores a nivel nacional e internacional (para el caso de Chile, ver Rojas Navarro, Rojas y Peña, 2018; Rojas Navarro, Rojas, Castillo-Sepúlveda y 
Schöngut-Grollmus, 2018). Sin embargo, reducir su potencialidad a esa única posible línea de acción resulta una caricaturización de los modos de articulación en que estos encuentros se producen. No hay que confundir el problema del sobrediagnóstico del TDAH, ni el fenómeno de su sobremedicalización, con el análisis que propongo acá, esto es, el seguir la pista a los modos concretos mediante los cuales cuerpos, subjetividades, fármacos, funciones psicológicas, materialidades, normas y disciplinas se imbrican, gestando la aparición de nuevos fenómenos, configurando nuevas entidades a partir de estos encuentros.

Para efectos de este análisis, cabe destacar que lo que interesa relevar son los fenómenos de producción social, y para ello centra su atención en los modos de interacción de materialidades diversas—tales como el fármaco y la niña o niño—en tanto como afectan y son afectados en su encuentro con otras materialidades. En ese sentido, lo central es poder destacar la materia

(...) no en términos de lo que es, sino que en términos de lo que hace: que asociaciones conduce, y que consecuencias se derivan de dichas interacciones. Consecuentemente, materialidades-cuerpos, objetos, órganos, especies y otras—no son tratadas como esencias definidas ontológicamente apriori, cada una ocupando su propio pedazo de tiempo y espacio, sino como relacionales, obteniendo una integridad y estatuto ontológico solamente a través de sus relaciones con otros igualmente contingentes y efímeros cuerpos, cosas e ideas. (Fox y Bale, 2018, p. 396)

La anterior se ve ilustrado en los eventos que compartiré a continuación. El primero de estos concierne al proceso de encuentro entre una niña y el fármaco, mientras que el segundo guarda relación con un niño. En ambos casos los nombres han sido anonimizados siguiendo los estandares éticos correspondientes a la investigación con niñas y niños. Los participantes de la praxiografía que originaron los eventos que compartiré dieron su asentimiento de participación de modo escrito, y sus padres y/o 
guardianes firmaron cartas de consentimiento, autorizando la participación de sus hijos y/o hijas. El trabajo etnográfico se desarrolló con alumnas y alumnos de quinto básico, y formó parte de una investigación doctoral. Por lo mismo, todos los aspectos éticos fueron aprobados por el comité de ética de la institución donde me encontraba afiliado al momento de la investigación. Así, la investigación se llevó adelante con todos los resguardos éticos, se informó a los participantes de las características del estudio, y participaron del mismo de forma libre y voluntaria.

El primero de estos eventos tiene relación con una niña llamada Angélica. Para cuando aconteció este evento, Angélica llevaba ya dos meses en tratamiento con psicoestimulantes, retomando así un tratamiento farmacológico que se había visto interrupido por las vacaciones de verano. La razón por la cual empezó su tratamiento se relaciona a sus bajas calificaciones, y al que su interés general hacia los contenidos académicos disminuyó considerablemente de acuerdo a sus padres y profesores, lo que también habría repercutido en su auto-estima. Durante una entrevista ella menciona que

cuando era chica me iba super bien, pero desde el año pasado me empezó a costar más que me importará lo que las profesoras decian en clases. Y al final cuando lograba poner atención ya no cachaba [entendía] nada porque estaban hablando de otras cosas, y yo me había quedado pegada en lo otro. Y es horrible sentirse así (Angélica). (Notas de campo, 2015)

Al preguntarle a Angélica respecto a qué sensación se refiere, ella continua del siguiente modo:

la sensación de quedarse atrás. Como darse cuenta que todas entendieron algo y yo no, y no porque no pueda, es porque estás en otra por un rato, como volada, y cuando despiertas tienes que ponerte al día pero estás siempre, siempre atrasada (Angélica). (Notas de campo, 2015) 
Algunas profesoras me comentan que desde que retomó el tratamiento tanto las notas de Angélica como su auto-percepción han mejorado. Sin embargo, la presencia de Angélica en la sala de clases sigue distando de aquel imaginario tradicionalmente asociado al niño disciplinado, así como tampoco parece encarnar las descripciones del niño alienado por el medicamento que esgrimen las teorías de la medicalización. Esto se evidencia un día en particular, a comienzos de junio, durante una clase de Lenguaje cuando—como en muchas otras ocasiones-Angélica se encuentra sentada en su banco. Pero en lugar de actuar como normalmente se esperaría que lo hicieraojos al frente, mirando al pizarrón, espalda recta y tomando notas—su cabeza está gacha, sus manos juegan con su pelo, el cual se encuentra amarrado en una larga trenza la que observa con detención. Durante el transcurso de la clase constantemente la encuentro así. Parece ausente, ensimismada, como si estuviese habitando un mundo propio hecho de sus ideas e intereses. Solo ocasionalmente deja de jugar con su pelo, toma el lápiz, y anota algo en el cuaderno. Lo único que parece romper este estado de habitar la sala de clases es cuando es reprendida por la profesora. Cuando esto ocurre Angélica se ve sorprendida. Se sienta derecha, y mira al frente. Sin embargo esto solamente dura unos minutos, tras los cuales su interés parece volver nuevamente a volcarse en su pelo.

Le pregunto respecto a lo anterior durante una entrevista. “¿Qué sientes tu que hacen los remedios?" le pregunto, a lo que agrego "te pregunto ya que varias veces pareciera que estás distraida, o como si no te importa mucho lo que los profesores dicen o hacen en la sala". Angélica me mira. Parece sorprendida por mi lectura de los hechos. "No, estás mal", me dice, "yo pongo como mucha, mucha atención casi todo el rato [tiempo]. Lo que pasa es que para mi es más facil poner atención sin mirar". Le pregunto a que se refiere con esto, qué es para ella "poner atención". Me responde diciendo: 
El año pasado en clases aprendimos que hay varias formas diferentes de aprender y de poner atención. Algunas niñas necesitan escribir, otras necesitan ver, y para mi es más fácil aprender si escucho. Como que mirar o no el pizarrón depende en verdad de la profesora, si es simpática, o si es muy retona y pesada. Como he tocado guitarra desde que era chiquitita, siempre me acostumbré a poner atención con los oidos (Angélica). (Notas de campo, 2015) Angélica parece extrañarse de mi dificultad en comprender cómo se despliegan los efectos de la medicación. Por lo mismo, me lo explica de otro modo:

No es tan raro. Es como que... cuando me tomo el remedio, y siento que empieza a funcionar, es más facil para mi elegir que escuchar y que no, y como... y poder seguir escuchando lo que yo quiera escuchar, en vez de escuchar otra cosa. En vez de ponerme a pensar en canciones o en lo que las cosas que hablo con las otras niñas, puedo escuchar lo que yo quiera escuchar (Angélica). (Notas de campo, 2015)

"Pero, ¿cómo sabes que está funcionando?" le pregunto. "Porque mi guatita [estómago] se siente diferente, y a veces también mis manos como que pican un poco, como si hormiguitas estuvieran caminando en mis manos. Siento eso a veces cuando me tomo el remedio”, responde. “¿Es incómodo o desagradable sentir eso? Me refiero a lo de tus manos y tu guatita, sentirlas diferente, que te pican y eso". Angélica reflexiona unos segundos, y luego responde:

En verdad no. A lo mejor al comienzo era un poco raro. Pero después aprendí que es así como funciona no más, y que no hay que asustarse ni nada por el estilo. Así se siente cuando te la tomas y está adentro de uno. Eso es todo (sonríe) (Angélica). (Notas de campo, 2015)

La reflexión de Angélica es interesante por muchas razones. Principalmente, debido a que sus ideas y acciones reflejan como el encuentro entre el psicoestimulante y su propio cuerpo ocurre. A partir de éste se produce la emergencia de una serie de 
nuevas capacidades para hacer y sentir, las cuales se estabilizan a través de la producción de una experiencia. Experiencia que logra cierta consistencia gracias a la producción de un entramado entre las diversas entidades presentes en la sala de clases, y entre su cuerpo y el medicamento. Este entramado no es una adición simple de diversos elementos. Por el contrario, un entramado posibilita una experiencia consistente en tanto conlleva una transformación, la emergencia de una nueva versión, una nueva entidad (Barad, 2007). La experiencia del encuentro entre Angélica y el medicamento en el nicho provisto por la sala de clases conlleva un proceso de profunda modificación que no la sitúa en una posición de docilidad. No es posible sostener que Angélica se encuentra simplemente coactada por el medicamento, ni que el proceso de modificaciones corporales que este produce, en última instancia, restringue o colapsa su integridad o coherencia. Sería demasiado simple reducir el análisis a contemplar esta interacción como una lucha de fuerzas, una liza entre dos formas de voluntad que pese a seguir diferentes trayectorias, se topan, y donde solamente una puede emerger victoriosa. Por el contrario, la experiencia de Angélica en la sala de clases llama a reflexionar respecto a los procesos mediante los cuales dos entidades dotadas de cierta consistencia-en tanto estables-se relacionan y coafectan. Respecto a cómo el encuentro de modos de materialidades-que-importan [forms of mattering] contingentemente produce formas de relacionalidad las cuales se fuerzan, negocian, y finalmente producen nuevas formas de constitución, de estabilidad (Savransky, 2016).

De este modo, el encuentro entre el psicoestimulante y Angélica no refleja los efectos de un vector de poder que forzaría un entrar en regla a la niña de acuerdo a estándares adultos. El juego de negociaciones que se establece con el fármaco posibilita la emergencia de otras cosas. En ese sentido, el despligue del fármaco en este caso no conlleva la posibilidad de reflejar en Angélica una disciplina escolar que cancelaría su subjetividad. Mas bien parece posiblitar una difracción de aquella 
experiencia escolar, permitiendo la posibilidad de emergencia de otra forma de subjetividad con la cual Angélica (con)vive armónicamente en tanto posibilita una forma de experiencia que es vivenciada con complacencia. El medicamento, así como también el encuentro con otros materialidades y discursividades de la sala de clase, operan como limitaciones "generosas" (Gomart, 2002) o “creativas" (Savransky, 2016), que más que estabilizarse en la fórmula de "la niña o la medicación", abren la pregunta respecto a las formas de experiencia que se generan productivamente a partir del encuentro de dos entidades que se encuentran, transforman, y generan algo nuevo a partir de aquel entramado farmacológico. En ese sentido, el fármaco en el encuentro con Angélica establece un proceso abierto, cuyo desenlace y estabilización depende de muchos elementos que van más allá-pero que incluyen y se ven gatillados por—la introducción y encuentro con dicha materialidad (Rojas Navarro y Vrecko, 2017).

Pero en tanto proceso abierto, y considerando las formas contingentes en que las entidades se encuentran, es que este proceso puede también tener otros desenlaces, el cual ejemplificaré con un segundo proceso de encuentro.

Este ocurrió una vez finalizado el recreo, cuando los niños volvían presurosos a clases. A medida que entran a la sala me fijo en Mateo. Su rostro está pálido, y refleja una expresión de cierta incomodidad y malestar. Se sienta en su puesto, y pese a mantenerse sentado, sus constantes intentos de re-acomodarse en la silla, así como también su respiración pesada y ciertos gestos tales como frotarse nerviosamente las manos una contra la otra, hacen pensar que algo no está bien. En ese momento entra Daniela, la profesora de Historia a la sala de clases. Pone sus cosas en el escritorio que se encuentra situado al costado derecho del pizarrón, saluda a los presentes, los hace callar, y comienza la clase. No más de 20 minutos más tarde-mientras la gran mayoría de los alumnos se encuentra trabajando en grupos, resolviendo una hoja con actividades-Daniela me hace señas para que vaya donde ella está. A medida que 
cruzo la sala, veo que discretamente apunta con su dedo a Mateo. Se le ve inquieto, incómodo. Se mueve y acomoda en la silla, y su cara sigue pálida.

Daniela se acerca a mí y suavemente susurra en mi oído "no se ve muy bien, ¿no crees?". Concuerdo con ella. Daniela reflexiona respecto a la situación por algunos segundos, y luego se agacha junto a Mateo. “¿Estás bien Mateo?, te veo un poco pálido" le dice cariñosamente. Él trata de sonreír, pero solamente logra esbozar una mueca. "En verdad no mucho", responde, "siento el pecho raro, como si el corazón latiera súper rápido". Daniela lo escucha con atención, y rápidamente le responde "bueno, eso no está nada de bien. Anda de inmediato a la enfermería". Luego se pone de pie, y llama a otro niño a quien pide que acompañe a Mateo para que no vaya solo. Cuando ambos niños salen de la sala le pregunto que piensa que le ocurre a Mateo. "Puede que sea por los remedios. A veces los niños reaccionan mal cuando los toman", dice. Le pregunto qué la hace sospechar que Mateo está tomando medicamentos. Daniela esboza una leve sonrisa, contrariada, como si hubiese notado que su respuesta no fue del todo satisfactoria, e inmediatamente agrega "es algo que estoy suponiendo, pero creo que es así ya que tuve que llenar un test de Conners respecto a Mateo hace unas semanas. Y si te fijaste, no se estaba comportando como siempre". Por esto, hace referencia a un modo previo de Mateo de habitar el espacio de la sala de clases, marcado por rasgos como la impulsividad y dificultad de seguir instrucciones de profesores o compañeros.

Cuando Mateo vuelve, hacia el final de la hora pedagógica, nos dice que se siente bastante mejor. Daniela le pregunta qué dijo la enfermera. "Me dijo que ella piensa que puede ser por el remedio que tomé en la mañana, y porque estuve jugando futbol en el recreo", responde Mateo, sin aparentemente darle mucha importancia al asunto, ya que parece más interesado en lo que están haciendo sus compañeros, quienes se alistan para salir a recreo. "Dame tu libreta de comunicaciones de todas formas. Le voy a escribir a tus papás para que sepan lo que pasó. No deberías tomar el remedio 
hasta que te vea un doctor, ¿bueno?”, dice Daniela. Mateo corre hasta la parte trasera de la sala, donde las mochilas están colgadas. Abre la suya, saca la libreta, y corre de vuelta a pasársela a la profesora, aprovechando de preguntar si puede salir a jugar. Daniela dice que si, pero que se quede tranquilo y no se esfuerce mucho. Luego, se va caminando a su escritorio a escribir la comunicación.

Acompaño a Mateo afuera y le pregunto cómo se siente. "Estoy mejor, pero me asusté mucho. Se sentía raro, como mi corazón latiendo muy rápido", me dice. "Te había pasado algo así antes?", pregunto, a lo que me responde "En verdad no. Había tomado el remedio antes este año, pero nunca me había sentido así... Ojalá no me pase de nuevo" dice en tono pensativo, mientras mira desde el segundo piso como otros niños juegan futbol en los patios de abajo. "Por qué crees que está vez pasó esto... como que funcionó mal", insisto. El responde:

No lo sé. Creo... no sé en verdad. Solo se que empecé a sentir mi corazón latir más fuerte, y como que me quedé concentrado en eso, en sentir eso, y me empecé a asustar y después ya no podía dejar de fijarme en eso. Fue súper raro, pero ya estoy mejor... ¿te importa si me voy a jugar? (Mateo). (Notas de campo, 2015)

Sonrió- mientras veo que se aleja corriendo a las escaleras para sumarse al partido que juegan sus compañeros.

Mateo produce otra clase de encuentro con la medicación, la cual es diferente a la enactada por Angélica. En el caso de Mateo, este encuentro parece producir efectos que se asemejan a los descritos por aquellos que se oponen al uso de psicofármacos. Sin embargo, cabe destacar el carácter original, particular en que se fragua este encuentro, en tanto que para producir una aproximación más rica de lo que ocurre en esta escena, resulta necesario ir más allá de la simple constatación de estos efectos displacenteros, y apreciar las formas de concatenación que se producen entre Mateo y el fármaco. Esto, en tanto que esta estabilización no es efecto únicamente de un 
fármaco cuya potencia colma la subjetividad del niño hasta saturarla. Más bien, aquello que emerge es un juego dinámico de negociaciones que posibilita la aparición de una nueva forma de habitar el mundo. Surge así una corporalización imbuida por las modificaciones químicas que conlleva el fármaco, pero que también establece tensiones y acomodaciones con otros cuerpos y materialidades a través de acciones y juegos las cuales dejan huellas, afectando-y afectándose por- esa corporalidad. Una nueva forma de subjetividad emerge a partir de la estabilización de estos entramados. Los efectos adversos no son entonces únicamente reducibles a la acción bioquímica de ese fármaco al impactar la biología de ese cuerpo. Son también relacionales, en tanto ese encuentro se genera y es orientado por encuentros de ese cuerpo con otras entidades las cuales producen, orientan, potencian y/o obscurecen dichos procesos.

La subjetividad del niño no debería entenderse como un dato a-priori, una esencia contenida en ese cuerpo que se ve contaminada por el fármaco. Por el contrario, es en ese proceso de encuentros que se produce la subjetividad, sostenida por el entramado de cuerpos, saberes y materialidades diversas que se encuentran en dicho nicho. Esto no quiere decir que esa subjetividad sea necesariamente una experiencia confortable. Es allí dónde dicho entramado es puesto en cuestionamiento no solamente por Mateo y su malestar, sino que también por otros fragmentos que componen la estabilización que permite el surgimiento de esa experiencia. Como se aprecia en el evento relatado, esa misma cualidad emergente de extrañeza y molestia conlleva a que este entramado se rompa, y que las entidades que se encontraron para dar pie a este modo de existencia sigan trayectorias divergentes.

\section{Conclusiones}

Los eventos relatados ilustran puntos centrales para pensar la subjetividad más allá de una comprensión que la reduce a una posesión interna de un individuo, o que la 
conceptualiza exclusivamente como una función inherente al mismo, incorporada "dentro" de un cuerpo que estaría esencialmente separado del mundo que lo rodea. Resulta necesario trascender esta forma de comprender lo subjetivo si es que efectivamente queremos aproximarnos a la complejidad del mundo, con su carácter profundamente relacional, procesal y dinámico. Tal como lo ilustran Angélica y Mateo, la subjetividad es un proceso en constante elaboración, la cual se genera a propósito de una serie de entramados, agrupamientos "ad hoc de diversos elementos, de materialidades vibrantes de todos los tipos", los cuales actúan como "confederaciones vivas, palpitantes, que logran funcionar pese a la persistente presencia de energías internas que tienden a la confusión" (Bennett, 2010. p. 24-25).

Si bien la cautela respecto al uso de psicofármacos resulta pertinente en tanto conlleva una serie de modificaciones corporales cuyos riesgos han sido advertidos anteriormente, resulta necesario abrir nuevas preguntas que generen y desarrollen reflexiones, teorías y métodos que rescaten la multiplicidad de entidades en juego, así como sus formas de vinculación y encuentro. Al abandonar la idea de que es únicamente un vector de poder el que opera-el fármaco coactando al niño o niña medicada—el análisis puede abrirse a pensar una "ecología de preguntas" que permita multiplicar los vectores (Savransky, 2014), rescatando así el carácter complejo y procesal de la subjetividad, la cual se estructura por medio de patrones que permiten estabilizar, de manera transitoria y frágil, elementos heterogéneos.

La subjetividad no sería entonces reductible a una propiedad interna-ya sea en jaque o bien constituida por un vector externo-sino que el producto de eventos que tendrían cierta consistencia en el tiempo, cierta estabilidad a partir del agrupamiento de elementos semiótico-materiales en un nicho determinado. Así, lo que surge es una consideración ontológica distinta para pensar el problema de los encuentros entre niñas, niños y psicoestimulantes, la cual se sostiene en la proposición de una "fundamental relacionalidad de toda materia: cuerpos, cosas y formaciones sociales" 
en tanto estos elementos heterogéneos solamente obtienen su apariencia de estabilidad "a través de sus relaciones con cuerpos, cosas, e ideas igualmente efímeras y contingentes", en donde "todo cuerpo, todo objeto, tiene una capacidad agencial de afectar" (Alldred \& Fox, 2017. p. 1163).

Estos entramados farmacológicos posibilitan la generación de una cierta coherencia que permite la aparición de una nueva versión de la niña medicada-en el caso de Angélica—la cual establece nuevas formas de relación con las entidades presentes en la sala de clase, y a partir de ello puede generar nuevas trayectorias las cuales dan cuenta de un modo nuevo de habitar ese nicho. Para Angélica, es a través de las limitaciones que se desprenden del usar la medicación, de las interacciones entre las materialidades del fármaco, su cuerpo y los otros, que se produce una nueva forma de subjetividad la cual resulta más diestra en construir un modo idiosincrático de habitar la sala de clases. Esta nueva versión de la subjetividad no refleja necesariamente la inscripción de la normalidad escolar ni una normativización de la conducta. Por el contrario, actúa creando efectos de diferencia, difractando esa imagen respecto a los modos y usos correctos en que atención y cuerpo deben ser utilizados en la sala de clases. Emerge entonces una versión "recalcitrante" de la subjetividad (Savransky, 2014).

Por el contrario, en el caso de Mateo, el entramado que se produce a partir de la introducción de la medicación no logra generar la coherencia, la consistencia necesaria para sostenerse. El efecto de la medicación, sus efectos materiales sobre el cuerpo, así como también la comprensión que de dichos efectos se realizan por parte de Mateo, son de tal fragilidad que tienden a la descomposición. No logra crearse una versión de la subjetividad que pueda encauzar este encuentro, sino que por el contrario dicho encuentro parece repeler este proceso de mutuo devenir. Frente a este, la profesora interrumpe las entidades que componen dicho ensamblaje. 
Multiplicar los vectores de análisis mediante la propuesta de una composición relacional y procesal de la subjetividad permite un entendimiento más rico de cómo las subjetividades, transitoria y contextualmente, emergen y se estabilizan. También permite interrogar de un modo diferente los efectos de los procesos de encuentro que niñas y niños establecen con otras entidades-como los psicofármacos-no reduciéndolos a procesos de lucha entre ellos, sino que entendiendo su carácter mutuamente constituyente y en constante re-elaboración, que pueden tener desenlaces dispares. Emerge así una noción no-esencialista de la subjetividad, la cual se considera parte y efecto de un mundo en el cual lo que realmente existe no son fenómenos discretos y terminados, sino que en constante elaboración y relación, lo cual dota a la subjetividad de cierta ambigüedad e inestabilidad que no debe ser resuelta, sino que entendida en tanto es la condición de posibilidad, de producción de aquello que podemos entender como la subjetividad (Despret, 2004).

\section{Referencias}

Alldred, P., \& Fox, N. J. (2017). Young bodies, power and resistance: a new materialist perspective. Journal of youth studies, 20(9), 1161-1175.

Barad, K. (2007). Meeting the universe halfway: quantum physics and the entanglements of matter and meaning. Durham \& London: Duke University Press.

Barad, K. (2003). Posthumanist performativity: toward an understanding of how matter comes to matter. Signs, 28(3), 801-831.

Bennett, J. (2010). Vibrant Matter. A political ecology of things. Durham \& London, UK: Duke University Press.

Bergey, M., Filippe, A., Conrad, P., \& Singh, I. (2018). Global Perspectives on ADHD: Social Dimensions of Diagnosis and Treatment in Sixteen Countries. Baltimore: Johns Hopkins University Press. 
Braidotti, R. (2016). Posthuman critical theory. En Banerji, D., \& Paranjape, M. (Eds), Critical posthumanism and planetary futures. New Delhi: Springer.

Conrad, P., \& Schneider, J. (1992) Deviance and medicalization: from badness to sickness. Philadelphia: Temple University Press.

Coole, D., \& Frost, S. (2010). New materialisms: ontology, agency, and politics. Durham \& London, UK: Duke University Press.

Despret, V. (2004). The body we care for: figures of anthropo-zoo-genesis. Body \& Society, 10(2-3), 111-134.

Faraone, S. \& Bianchi, E. (eds.) (2018). Medicalización, salud mental e infancias. Perspectivas y debates desde las ciencias sociales y el sur de América Latina. Buenos Aires, Argentina: Editorial Teseo.

Fox, N. J., \& Bale, C. (2018). Bodies, pornography and the circumscription of sexuality: a new materialist study of young people's sexual practices. Sexualities, 21(3), 393-409.

Gomart, E. (2002). Towards generous constraint: freedom and coercion in a French addiction treatment. Sociology of Health \& IIIness, 24(5), 517-549.

Haraway, D. (1992). The promises of monsters: a regenerative politics for inappropriate/d others. En Grossberg, L., Nelson, C., \& Treinchler, P. (eds), Cultural studies. New York: Routledge Press.

Harwood, V. (2006). Diagnosing 'disorderly' children. A critique of behavior disorder discourses. New York: Routledge Press.

Latour, B. (2004). Whose cosmos, which cosmopolitics?: comments on the peace terms of Ulrich Beck. Common Knowledge, 10(3), 450-462.

Marchand, J. (2018). Non-human agency. En Braidotti, R. \& Hlavajova, M. Posthuman Glossary (pp. 292-295). London, New York: Bloomsbury. 
Mol, A. (1999). Ontological politics. A word and some questions. En Law, J. \& Hassard, J. (eds). Actor network theory and after. UK: Blackwell Publishers/The Sociological Review.

Mol, A. (2002). The Body Multiple: Ontology in Medical Practice. Durham: Duke University Press.

Rojas, P. (2017). Después de los estudios sociales de ciencia y tecnología: recomponiendo lo psicológico ante la bifurcacón de la naturaleza. Athenea Digital, 17(3), 225-244.

Rojas Navarro, S. \& Vrecko, S. (2017). Pharmaceutical entanglements: an analysis of the multiple determinants of ADHD medication effects in a Chilean school. International journal of qualitative studies on health and well-being, 12:sup1.

Rojas Navarro, S., Rojas, P. \& Peña, M. (2018). From problematic children to problematic diagnosis. The paradoxical trajectories of child and adolescent ADHD in Chile. En Bergey, M. R., Filippe, A. M., Conrad, P. y Singh, I. (eds.). Global perspectives on ADHD. Social dimensions of diagnosis and treatments in sixteen countries (pp. 310-321). Baltimore: Johns Hopkins University Press

Rojas Navarro, S., Rojas, P., Castillo-Sepúlveda, J. \& Schöngut-Grollmus, N. (2018). Reensamblando la medicalización: hacia una pluralización de las explicaciones del TDAH en Chile. En Faraone, S. y Bianchi, E. (eds.). Medicalización, salud mental e infancias. Perspectivas y debates desde las ciencias sociales en Argentina y el sur de América Latina (pp. 335-368). Buenos Aires, Argentina: Editorial Teseo.

Savransky, M. (2014). Of recalcitrant subjects. Culture, Theory and Critique, 55(1), 96113.

Savransky, M. (2016). The adventure of relevance. An ethics of social inquiry. London: Palgrave Macmillan.

Stengers, I. (2011) Cosmopolitics I. Minneapolis, MN: University of Minnesota Press. 
This article was funded by CONICYT PIA CIE160007 // La escritura de este artículo ha sido posible gracias al financiamiento del proyecto CONICYT PIA CIE 160007.

\section{Formato de citación}

Rojas, S. (2018). Nuevas subjetividades: una aproximación posthumanista y material a los procesos de encuentro entre niñas, niños y psicoestimulantes. Psicología, Conocimiento y Sociedad, 8(2), 170-193. doi: http://dx.doi.org/10.26864/PCS.v8.n2.9 